\title{
Algorithmic Approach to Eccentricities, Diameters and Radii of Graphs using DFS
}

\author{
Ishwar Baidari \\ Asst Professor \\ Dept of Computer Science \\ Karnatak University, Dharwad
}

\author{
Ravi Roogi \\ Research Scholar \\ Dept of Computer Science \\ Karnatak University, Dharwad
}

\author{
Shridevi Shinde \\ Research Scholar \\ Dept of Computer Science \\ Karnatak University,Dharwad
}

\begin{abstract}
Let $G=(V, E)$ be a graph. The distance $d(u, v)$ between two nodes $u$ and $v$ is the length of the shortest path between them. The eccentricity $E(v)$ of a graph vertex $v$ in connected graph $G$ is the maximum distance between $v$ and any other vertex $u$

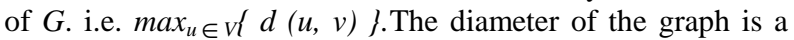
graph the longest shortest path between any two graph vertices $(u, v)$ of a graph i.e. $\operatorname{Diam}(G)=\max \{E(v)\} v$ $\in V\}$. The minimum eccentricity of a graph is radius i.e. Rad $(G)=\min \{E(v) / v \in V\}$. In this paper we propose algorithms for finding eccentricity diameter and radius of a tree using DFS.
\end{abstract}

Keywords: Eccentricity, Radius, Distance, Diameter, and Graph.

\section{INTRODUCTION}

A graph is a collection of points and lines connecting some of them. The points of a graph are most commonly known as graph vertices. Similarly the lines connecting the vertices of a graph are most commonly known as graph edges. Formally we can define a graph as a graph $G$ is a pair of sets $V$ and $E$ together with a function. $f: E \rightarrow V \times V$ The elements of $V$ are vertices and the elements of $E$ are edges. Connections between the points come in two forms those that are nondirectional and those that have an implicit direction are undirected and directed respectively.

In a graph theory a tree is connected acyclic graph stated otherwise trees and graph are undirected. A tree is called a rooted tree if one vertex has been designated the root in which case the edges have a natural orientation towards or away from the root.

\section{Eccentricity}

The concept of eccentricity is fundamental in graph theory. In this paper we are designing an algorithm for finding eccentricity of a tree. Tree is connected graph with no cycles. In an undirected tree a leaf is a vertex of degree 1 . Some basic properties of trees are:

1. Every tree with at least one edge has at least two leaves. If the minimum degree of a graph is at least 2 , then that graph must contain a cycle.

2. Every tree on $n$ vertices has exactly $n$-ledges.

1.1 Eccentricity of a tree: the eccentricity of a vertex $v$ in a graph $G$. Denoted $e c c(v)$, is the distance from $v$ to a vertex farthest from $v$ that is

$$
e c c(v)=\max _{x \in V G}\{d(v, x)\} .
$$

A central vertex of a graph is a vertex with minimum eccentricity. We begin with some existing [1] preliminary results concerning the eccentricity of vertices in a tree.

Lemma 1: Let $T$ be a tree with at least three vertices

a) If $v$ is a leaf of $T$ and $w$ is its neighbor, then

$$
e c c(v)=e c c(w)+1 .
$$

b) If $v$ is a central vertex of $T$, then

$$
\operatorname{deg}(v)=\geq 2 .
$$

Lemma 2: Let $v$ and $w$ be two vertices in a tree $T$ such that $w$ is of maximum, distance from $v($ i.e. ecc $(v)=d(v, w))$ then $w$ is a leaf.

Lemma 3: Let $T$ be a tree with at least three vertices, and let $T^{*}$ be the subtree of $T$ obtained by deleting from $T$ all its leaves. If $v$ is a vertex of $T^{*}$, then $\operatorname{ecc}_{T}(v)=\operatorname{ecc} T^{*}(V)+1$.

Let $T$ be tree

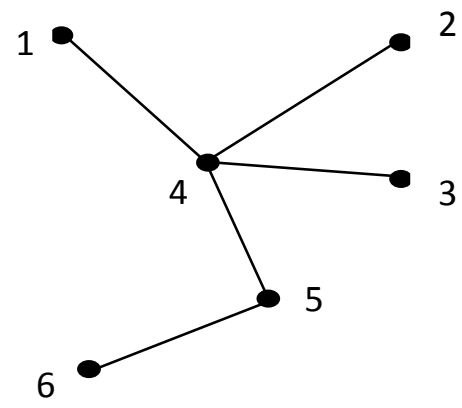

Consider the above tree, and then the eccentricity of each vertex in the tree is given below.

$$
\begin{aligned}
& E(1)=3 \\
& E(2)=3 \\
& E(3)=3 \\
& E(4)=2 \\
& E(5)=2 \\
& E(6)=3
\end{aligned}
$$

Algorithm: Eccentricity, Diameter and Radius of a tree Input: $n$ - number of nodes Cost[50][50] - adjacency matrix

Output: Eccentricity, Diameter and Radius 
1. Input the number of nodes and adjacency matrix

2. for $i \leftarrow 0$ to $n-1$

$$
\begin{aligned}
& \text { for } j \leftarrow 0 \text { to } n-1 \\
& \qquad D[i][j]=\operatorname{cost}[i][j] \\
& \text { End } j \\
& \text { End } i
\end{aligned}
$$

3. To find the shortest distance

$$
\begin{aligned}
& \text { for } k \leftarrow 0 \text { to } n-1 \\
& \quad \text { for } i \leftarrow 0 \text { to } n-1 \\
& \quad \text { for } j \leftarrow 0 \text { to } n-1 \\
& D[i][j]=\min 1(D[i][j], D[j][k], D[k][i]) \\
& \quad \text { End } j
\end{aligned}
$$

$$
\text { End } i
$$

End $k$

\section{To find eccentricity}

$$
\text { for } i \leftarrow 0 \text { to } n-1
$$

Initialize $\max =D[i][0]$

$$
\text { for } j \leftarrow 0 \text { to } n-1
$$

if $D[i][j]>\max$

$\max =D[i][j]$

$$
\text { End } j
$$

$E[i]=\max$

End $i$

2. Diameter: Determining the diameter of a graph is a fundamental seemingly quite time consuming operation but we are restricted it to tree. Recall that the eccentricity of vertex $x . e c c(x)=\max _{y \in \mathrm{V}} \mathrm{d}(\mathrm{x}, \mathrm{y})$, where $\mathrm{d}(\mathrm{x}, \mathrm{y})$ denotes the distance between $x$ and $y$ : the diameter of $G$ equals the maximum eccentricity of any vertex in $V$

Let $G$ be a graph and $v$ be a vertex of $G$. The diameter of $G$ is the maximum eccentricity among the vertices of $e$.

Thus, diameter $(G)=\max \{e(v): v$ in $V(G)\}$

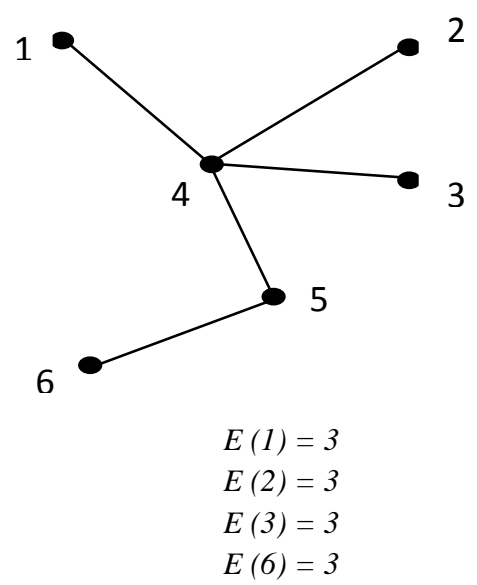

Diameter is 3 .

Let us now consider the existing properties [1] to help us to find the diameter of tree.
2.1 Fact: Suppose that $S P_{T}\left(v_{l}, v_{2}\right)$ is a diameter of $T$ and $r$ is a vertex on the diameter. For any vertex $x$,

$d_{T}(x, r) \leq \max \left\{d_{T}\left(r, v_{1}\right), d_{T}\left(r, v_{2}\right)\right\}$.

3.2 Lemma: Let $r$ be any vertex in a tree $T$, If $v$ is the farthest vertex to $r$, the eccentricity of $v$ is the diameter of $T$.

Algorithm: Diameter

5. To find Diameter

Initialize min and $\max =e[0]$

for $i \leftarrow 0$ to $n-1$

if $e[i]>\max$

$\max =e[i]$

else if $e[i]<\min$

$\min =e[i]$

End $i$

Diameter $=\max$.

3. Radius: The minimum eccentricity of all points in a graph is called the radius $r(G)$ of the graph. The radius can be obtained from a diameter.

The radius of $\mathrm{G}$ is the minimum eccentricity among the vertices of $\mathrm{G}$. Therefore radius $(G)=\min \{e(v) / v$ in $V(G)\}$

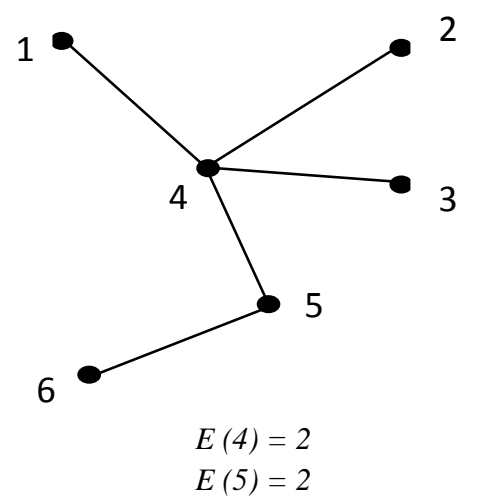

Radius of the graph is 2 .

Suppose that $p=S P_{T}\left(v_{l}, v_{2}\right)$ is a diameter. Starting at $V_{l}$ and travelling along the path $p$. We compute the distance $d_{T}\left(u_{l}, v_{l}\right)$ for each vertex $u$ on the path. Let $u_{1}$ be the last encountered vertex Such that $d_{T}\left(u_{1}, v_{1}\right) \leq 1 / 2 w(p)$ and $u_{2}$ be the next vertex to $u_{l}$ as shown in the below fig [1]

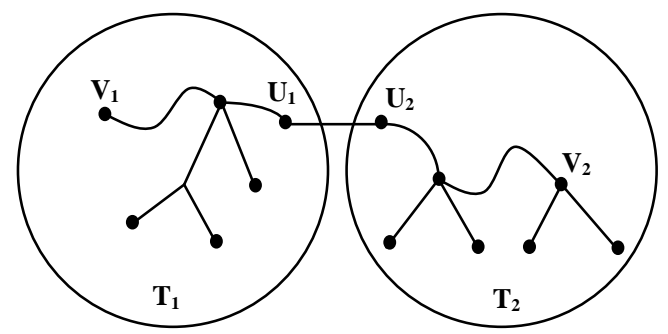

\subsection{Algorithm: Radius}
6. To find the radiu
for $i \leftarrow 0$ to $n-1$
if $e[i]>\max$
$\max =e[i]$
else if $e[i]<\min$
$\min =e[i]$ end $i$
Radius $=\min$

Initialize min and $\max =e[0]$ 
5. We proposed another program which find the eccentricity diameter and radii of graphs using DFS. We implemented it through linked list

\section{1}

\#include<stdio.h>

\#define FALSE 0

\# define TRUE 1

\# define SIZE 15

Typedef struc node

\{

int inf;

int weight;

struct node *link;

\}node;

typedef struct table

\{

int visit;

char data;

\}table; node *nodeptr;

table *tab[SIZE]

int $\max =0, \mathrm{n}, \mathrm{e}[50], \mathrm{I}, \mathrm{j}$;

Void dfs(int);

Void create(int)

Void main()

\{

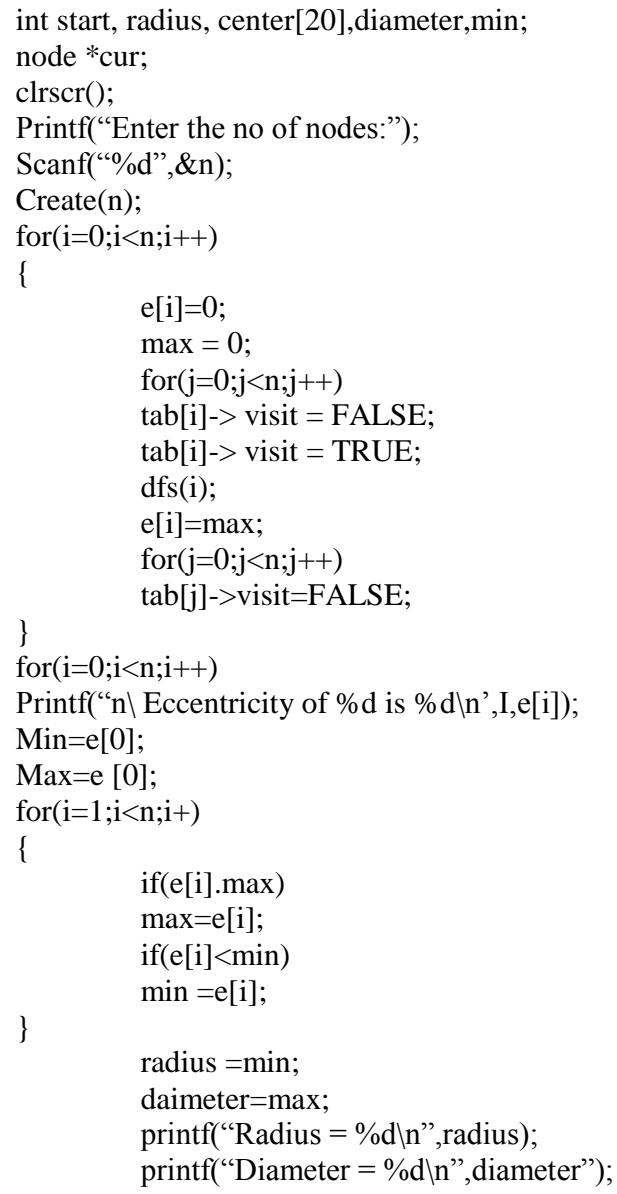

Void create(int $\mathrm{n}$ )

Node *new1,*temp;

printf("Enter the elements of the matrix below: $\mid n$ "); for $(\mathrm{i}=0 ; \mathrm{i}<\mathrm{n} ; \mathrm{i}++)$

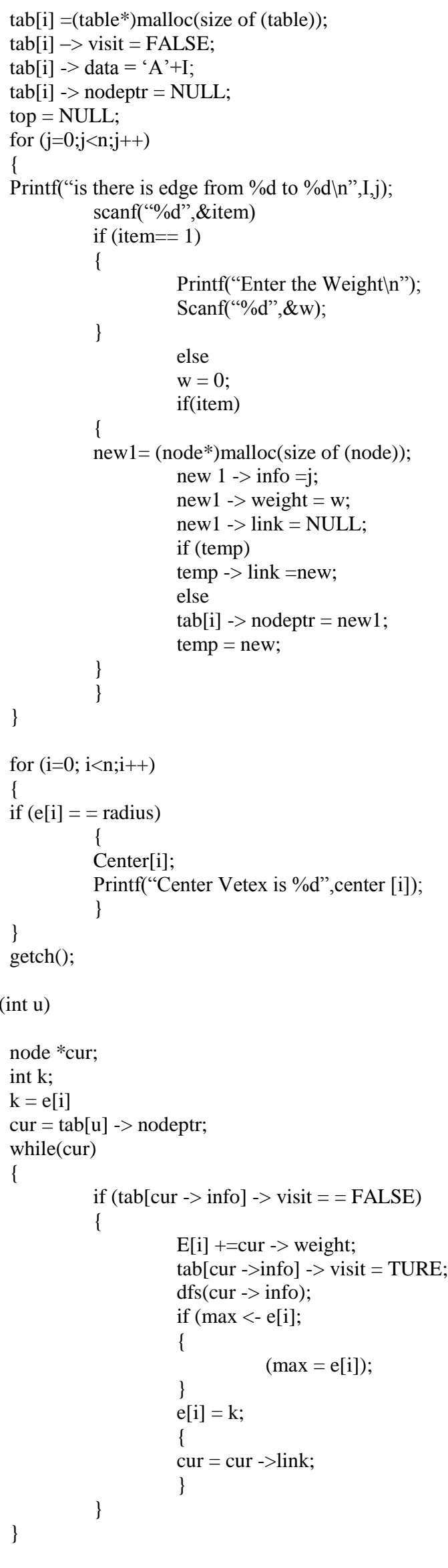




\section{REFERENCES}

[1]. A note on Eccentricities, diameters, and radii Bang Ye Wu Kun-Mao Chao

[2]. Alan Gibbons, Algorithmic Graph Theory. Cambridge University Press. 1999

[3]. Lich - Hsing Hsu and Cheng- kuan Lin Graph Theory and Interconnection Networks.CRC Press 2009.

[4]. Alfred V Aho, John E, Hopcroft and Jeffrey D. Ullman Data structures and Algorithms. Pearson Education 2006.

[5]. Thomas H Cormen Charles E Leiserson and Ronald L, Rivest. Algorithms PHI 2001.
[6]. Geir Agnarsson, Raymond Greenlaw. Graph Theory Modeling Applications and Algorithms.

[7]. Dieter Jungnickel Graphs Networks and Algorithms Springer 2006.

[8]. E COCKAYNE and S.GODDMAN and .HEDETINIEMI. A Liner Algorithm for the Domination Number of a Tree

[9]. B.S.Panda and D.Pradhan. Locally Connected Spanning Trees in Cographs, Complements of Bipartite Graph and Doubly Chordal Graph.

[10].Gary chartarand, Ortrud R Oellermann, Applied and Algorithmic Graph Theory Mc Graw-Hill Inc 1993 